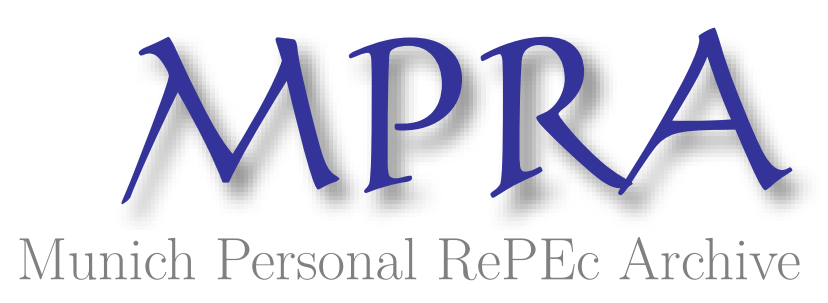

\title{
Social choice and the topology of spaces of preferences
}

Chichilnisky, Graciela

1980

Online at https://mpra.ub.uni-muenchen.de/8006/

MPRA Paper No. 8006, posted 31 Mar 2008 05:40 UTC 


\title{
Social Choice and the Topology of Spaces of Preferences
}

\author{
Graciela Chichilnisky*
}

Department of Economics, Columbia University, Netw York 10027

\section{INTRODUCTION}

Social choice theory is concerned with providing a rationale for social decisions when individuals have diverse opinions. Voting is an obvious way in which societies aggregate individual preferences to obtain social ones. The procedure of voting registers individual comparisons between alternatives, called ordinal preferences, rather than the intensities of preferences among these alternatives, called cardinal preferences, and this is one source of so-called paradoxes of social choice. ${ }^{1}$

The possibility that majority voting may be in contradiction to some criteria of rationality of preferences, such as the property of transitivity of the social choice, has been known for a long time, and is usually called the "paradox of voting" or the Condorcet effect, published first in 1785 by Condorcet in a book on the theory of elections. ${ }^{2}$ More recently the general theory of elections became a fertile field of research, beginning with the work of Black published in 1948 and 1949, and Arrow's 1951 monograph. Arrow stated in a formal way a set of seemingly reasonable criteria for social choice and proved that they are inconsistent. ${ }^{3}$

* This research was supported in part by the ONR Project on Efficiency of Decision Making in Economic Systems, Harvard University. I am grateful to K. Arrow, D. Anderson, H. Halkin, M. Hirsch, F. Peterson, and R. Willig for their valuable comments and suggestions.

'In this context, it is usual to call a "paradox" a contradiction within a set of seemingly reasonable axioms, or properties, of the rule that relates the individual preferences to the social ones.

${ }^{2}$ For a historical discussion of formal theories of social choice, see [2]. The Condorcet paradox can be sumarized as follows. Let there be three choices denoted $A, B$, and $C$, and three voters, denoted $x, y$, and $z$. If $x$ 's preferences among the choices are (in descending order) $A, B$, and $C, y^{\prime}$ s preferences $C, A$, and $B$, and $z^{\prime}$ s preferences $B, C$, and $A$, then a majority prefer $A$ to $B, B$ to $C$, and also $C$ to $A$, so that the social choice rule cannot be transitive. This cyclical element in the Condorcet paradox is indicative of a topological problem.

3 Arrow's conditions on the social decision rule are: (1) For any possible set of individual preference orderings, there should be defined a social preference ordering (connected and transitive) that governs social choices. (2) If everybody prefers alternative 
It is the purpose of this paper to study a paradox of social choice and to show that it arises, in part, because of the topological structure of spaces of ordinal preferences.

The problem can be summarized as follows. When intensities of comparisons among alternatives are considered, each (cardinal) preference can be represented by a unique utility or welfarc function, a real-valued function defined on the space of available choices; higher values correspond to more preferable choiccs. In this case, the spaces of cardinal preferences are function spaces, which are linear, and hence topologically trivial. This cardinalist approach goes back to Bernoulli (1730) [2]. The linearity of these spaces of preferences is neccssary for defining certain critcria of aggregation based on sums or on averages of the individual utility functions. Such criteria were introduced for normative reasons, such as impartiality of the social choices. ${ }^{4}$ For example, in 1945 Vickrey applied to social choice the Von NeumannMorgenstern theory of utility for risk bearing, to yield a criterion of aggregating individual preferences into social ones, that consisted of averaging individual utilities. Even before, Bentham (1780) and Edgeworth (1881) proposed adding up the utilities of the individuals to obtain the social welfare function.

However, when ordinal preferences are considered, linear or locally linear structures are not available, and this is a source of problems of aggregation of individual preferences in social choice theory. For instance normative criteria of aggregation of individual preferences into social ones that rely on addition or averaging are not well defined for ordinal preferences. More in general, as we show in this paper, spaces of ordinal preferences are not concontractible, and their topological structure produces paradoxes of social choice. We discuss this next.

While a cardinal preference is a utility function, ordinal preference can actually be thought of as an equivalence class of such functions. ${ }^{5}$ The equivalence relation $(\sim)$ is given by $u_{1} \sim u_{2}$ iff for all $x$ in the choice space, the preferred set of $x$ according to $u_{1}$ (i.e., the set $\left.\left\{y: u_{1}(y) \geqslant u_{1}(x)\right\}\right)$ is the same as the

$A$ to alternative $B$, then society must have the same preference (Pareto optimality). (3) The social choice made from any set of alternatives should depend on the individual preferences among the alternative of this set, and be independent of the individual preferences outside the set (independence of irrelevant alternatives). (4) The social procedure should not be dictatorial, in the sense that there is no one whose preference prevails regardless of the preferences of others.

+The criterion of impartiality was interpreted to mean that the social rule should choose as if it were equally likely to have any position in society. One decision would then be preferred to another if the expected utility of the first were higher. Since all positions are assumed to be equally likely, expected utility (i.e., Von NeumannMorgenstern utility function that explains behavior in risk bearing) is the same as the average utilities of the individuals.

- For general conditions under which an ordinal preference relation can be represented by a utility function, see, for instance, Debreu [4]. 
preferred set of $x$ according to $u_{2}$. Under certain conditions it suffices, in order that $u_{1}$ and $u_{2}$ be equivalent, that for each $x$ the hypersurfaces of $u_{1}$ and of $u_{2}$ that contain $x$ be equal, and that both $u_{1}$ and $u_{2}$ increase at the same side of them at $x$. These hypersurfaces are called indifference surfaces because the individual is indifferent between any two of its points.

Even though the space of utility equations is linear, the quotient space under $\sim$ is not lincar under the inherited sum, because $\sim$ does not respect the linearity of the space.

If, for instance the utility function is $C^{k}(k \geqslant 2)$, and the space of choices is a smooth manifold, then under certain conditions an ordinal preference is uniquely described by the set of all the hypersurfaces of the function and an orientation, and thus is uniquely represented by a globally integrable oriented $C^{k-1}$ codimension-1 foliation. Such spaces of ordinal preferences can then be thought of as spaces of globally integrable oriented codimension-1 foliations of the choice space. For a more detailed discussion of these points see Chichilnisky [3].

More in general, ordinal preferences are defined sometimes by a unit vector field, such as, for instance, the unit vector ficld normal to the indifference surfaces at each point. Such a vector field indicates the most preferred direction. This was, in fact, Antonelli's definition in 1886 [4], in which the vector field is only assurned to be locally integrable, so that the associated oriented foliation need not be globally integrable. For further discussion of smooth preferences and their properties, see, for instance, Debreu [4]. In the latter formulation, which is the one used in this paper, spaces of ordinal preferences are, then, spaces of oriented codimension-1 foliations of the choice space.

If one proposes to define aggregation rules based on desired normative properties of the social choice rule, such as, for instance, the addition rules of Bentham and Edgeworth, or the Von Neumann-Morgenstern-Vickrey averaging rule, a natural question is whether any linear structure can be given to these spaces of ordinal preferences, such as, for instance, spaces of oriented codimension- 1 foliations of the manifolds of choices without singularities, so as to be consistent with a natural topology. More generally, can such a space of preferences admit any continuous averaging rule for aggregating preferences, or is it contractible?

It turns out that the answer to these questions, a negative one, is contained as a special case of a new paradox of social choice, which, however, has certain elements in common with the Condorcet and Arrow paradoxes. We prove that the following three axioms on the rule $\phi$ that assigns to an $n$-tuple of ordinal preferences (the voters') the social preference are inconsistent:

(a) $\phi$ is continuous;

(b) $\phi$ respects anonymity of the voters, i.e., $\phi$ is invariant under permutation of its arguments; and 
(c) $\phi$ respects unanimity, i,e., if all the voters have the same preference, the social preference is the same as theirs.

This paradox is different in nature from existing social choice paradoxes: this result is topological while existing work in the area is algebraic or combinatorial. ${ }^{6}$

Two of the axioms ((b) and (c)) are similar to Arrow's in some respects; axiom (c) is strictly weaker than his Pareto condition. The first axiom, (a), is the main difference with Arrow's result: his axiom of independence of irrelevant alternatives (which effectively reduces the problem to a choice set with three elements) is not required for our result, while continuity of the map that assigns a social preference to the set of individuals is required here.

This requirement of continuity of $\phi$ can be considered natural. It arises from either (1) the desirability of the existence of sufficient statistics for measuring individual preferences under imperfect information, since, when $\phi$ is continuous, further statistical information about individual preferences that approach their real ones can be expected to get us closer to the appropriate social preference; or (2) a basic form of stability of socicty's choices with respect to small changes in individual preferences. Both these arguments are reminiscent of questions of "structural stability" of society's rules.

\section{A Social Choice Paradox: Inconsistency of the Properties of Anonymity, Respect of UNanimity, and Stability of Constitutions}

We assume that there are $k(k>1)$ individuals in society, each with preferences over a space of all possible choices available, called the choice space, denoted $M . M$ is assumed to be a manifold of dimension at least 2. A typical example in economics is the unit ball in $R^{n}$. Another typical example in economics is the interior of the positive quadrant of $R^{n}$, denoted $R^{n+}$. Both these cases are considered here as models for $M{ }^{7}$

Each individual preference is represented, as discussed above, by a $C^{r}(r \geqslant 1)$ codimension-1 foliation ${ }^{8}$ of $M$ which is transversally oriented. Thus, the space

"It has been proved that certain special subspaces of these spaces of preferences can actually be given (infinite dimensional) manifold structures; under certain conditions there are even isomorphic to convex subsets of a linear topological space; see Chichilnisky [3]. Such subspaces do admit averaging functions. The fact that the whole space does not admit such a function, i.e., that a certain function defined on a subspace does not admit an adequate extension to the whole space, describes a topological property of the space. This is discussed in Section 2.

${ }^{\top} M$ can also be a manifold with boundary, such as the (closed) unit ball in $R^{n}$. In that case one has to specify that the foliations be defined in a neighborhood of the (closed) unit ball. The results given here also apply for the closed unit ball in $R^{n}$.

"A $C^{\prime}$ codimension-1 foliation is given by a $C^{\gamma}$ locally integrable hyperplane field. 
of preferences, denoted $P$, is a space of $C^{+}$codimension- 1 transversally oriented foliations of the choice space $M$. We also use here other spaces of preferences which appear frequently in economics. One is the subspace of $P$ consisting of globally integrable foliations in $P$. This space, denoted $Q$, is a quotient space of a function space,

$$
Q=F / \sim
$$

where $F$ is the space of all real-valued $C^{++1}$ functions on $M$, and $\sim$ is the equivalence relation defined in the Introduction. Another subspace frequently used in economics is $R$, the space of all convex preferences of $Q$, i.e,, the space of preferences $p$ in $Q$ which satisfy, for all $x$ in $M$, that set $\{y: u(y) \geqslant u(x)\}$ is convex, where $u$ is any function in the equivalence class that defines $p$. The results given here apply to the spaces $Q$ and $R$ as well. A constitution $\phi$ is a rule that assigns, to a $k$-tuple of individual preferences, another, the social preference. Hence, $\phi$ is a map

$$
\phi: \begin{gathered}
k \text {-times } \\
\phi x \cdots x P \rightarrow P .
\end{gathered}
$$

Note that the points in the choice space $M$ may represent choices of all the individuals: an individual may have preferences not only over his/her choices but also over the choices of others. In this case, the dimension of $M$ is a multiple of $k$.

We work here with foliations without singularities; i.e., the vector fields defining these foliations do not have zeros in the interior of $M$. This corresponds to a property of nonsatiation of the individual's preferences in $M$. When the space of choices $M$ is a manifold with boundary, such as the closed unit ball, then the singularities will lie in the boundary of $M$. An extension of the results to foliations with singularities is given elsewhere. In the latter case the conditions needed are more stringent: (c) is replaced by a Pareto-type condition.

Since we study stability of the constitution, which refers to continuity of the map $\phi$, we now discuss the topology of the spaces of preferences. The space $P$ has a natural topology induced by the inclusion $P C V=\left\{C^{r}\right.$ vector fields of $M$ \}. If $M$ is compact, the $C^{r}$ sup norm induces a natural topology $\tau$ on $P$.

We consider a case where $M$ is the closed ball in $R^{n}$, a manifold with boundary; then the usual definition of a $C^{r}$ preference, say, in $Q$, would be an equivalence class of $C^{++1}$ functions which admit an extension to a neighborhood of $M . Q$ inherits a topology from that of the space of functions $F$, since $Q=F / \sim$. If $F$ is a space of $C^{r+1}$ real-valued functions on $M$ and $F$ is given a $C^{++1}$ sup norm, the preferences are gradient fields of functions in $F$ and thus the inherited topology on $Q$ is equivalent to $T$.

When the choice space is the interior of $R^{n+}$, and thus unbounded, a case frequently appearing in economics, other topologies are needed since the sup 
norms may not be well defined. In Chichilnisky [3], a Sobolev-Hilbert manifold structure denoted $\mathrm{H}^{s}$ is given to certain subspaces of preferences. The Sobolev theorem, which asserts that $H^{a} \mathrm{C} C^{r}$ if $s>n / 2+r$, does not give continuity of the inclusion $\mathrm{H}^{3} \mathrm{C} \mathrm{Cr}$ if the base manifold is not compact. However, this inclusion is locally continuous, locally in the sense of restricting the $H^{*}$ space of preferences to be defined on a compact neighborhood of a point in the choice space. Such local continuity is all that is needed to extend our results to $M=R^{n+}$, as seen in the proof of Theorem 1. See also [3] for more details.

We now assume that $P(Q$ or $R)$ is given one of the topologies above: either $\tau$, or a Sobolev $H^{\prime}$ norm with $s>n / 2+r$ if $M$ is unbounded.

\section{Theorem 1. There does not exist a constitution}

$$
\phi: \stackrel{k \text {-times }}{P x \cdots x P \rightarrow P}
$$

satisfying:

(1) stability, i.e., $\phi$ is continuous;

(2) anonymity, i.e., $\phi\left(p_{1}, \ldots, p_{k}\right)-\phi\left(p_{\sigma}(1), \ldots, p_{\sigma(k)}\right)$; for any permutation $\sigma$ of $\{1, \ldots, k\}$; and

(3) respect of unanimity, i.e., $\phi(p, \ldots, p)-p$, for all $p \in P$.

Proof. Let $x$ be a choice in the space $M$, and let $S^{n-1}$ be the unit sphere in $R^{n}$. Given a chart for $M$ at $x$, each preference $p$ in $P$ determines uniquely a point $z$ in $S^{*-1}$ given by the normal unit vector $v$ of the indifference surface i.c., the leaf of $p$, at $x$, in the orienting direction (see Fig. 1). This determines a map $\Gamma$ from $P$ to $S^{n-1}$ which is continuous by the choice of topology of $P$. Also, if $z \in S^{n-1}$, there is some preference in $P$ that projects onto $z$ under $\Gamma$. For instance, consider a preference $p_{z}$ in $P$ such that restricted to some neighborhood of $x$ is a foliation orthogonal to the vector $z$ at $p$, and oriented by $z$

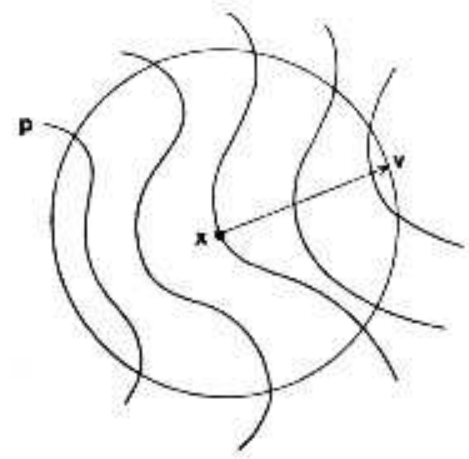




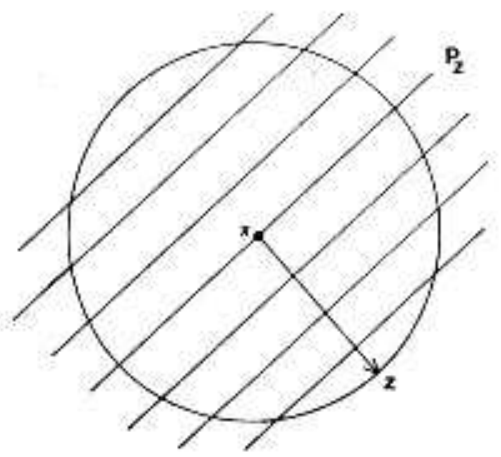

Figure 2

(sec Fig. 2). Such a foliation $p_{z}$ can always be constructed: since $M$ is either a ball or else the positive orthant of $R^{n}$, the map $\lambda$ defined by $\lambda(z)=p_{z}$ can also be chosen to be continuous from $S^{n-1}$ to $P$. Also, the composition map $\Gamma \circ \lambda$ is the identity map on $S^{n-1}$.

Assume now that there exists a map $\phi: P \times \cdots \times P \rightarrow P$ satisfying (1), (2), and (3). We shall show that this implies the existence of a map $\psi: S^{n-1} \times$ $\cdots \times S^{n-1} \rightarrow S^{n-1}$ satisfying conditions analogous to (1), (2), and (3).

Let \& be defined by the diagram

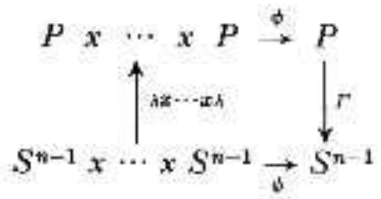

That is, $\psi\left(z_{1}, \ldots, z_{n}\right)=\Gamma\left(\phi\left(\lambda\left(z_{1}\right), \ldots, \lambda\left(z_{n}\right)\right)\right.$. Then $\psi$ is continuous, since $\Gamma$ and $\lambda$ are, and $\phi$ satisfies (1). It is easy to check that $\psi$ satisfies conditions (2) and (3) also, by its definition. However, as we shall now show, such a map $\psi$ cannot exist. We consider first the case of two individuals, i,e., $k-2$. We now give a heuristic argument for a special case of this nonexistence result which illustrates the general result. The complete proof is given following this illustration.

Let $\psi: S^{1} \times S^{1} \rightarrow S^{1}$ be a smooth function satisfying (1), (2), and (3). Let $D$ be the diagonal in $S^{1} \times S^{1}$, i.e., $D-\left\{(\alpha, \beta)\right.$ in $S^{1} \times S^{1}$ with $\left.\alpha=\beta\right\}$. (See Fig. 3 for an illustration.) Let $(\alpha, \alpha)$ be a point in $D$. At $(\alpha, \alpha)$ the determinant of the Jacobian matrix $J\left(\psi_{i} D\right)(\alpha, \alpha)+0$ and the index $\bmod 2$ of $\psi_{i} D$ at $(\alpha, \alpha)$ must be 1 . This follows from the condition of respect unanimity (3),

i.e.,

$$
\psi(D(\beta, \beta)=\beta,
$$

$$
\psi \circ \operatorname{Incl}_{D}-\operatorname{Id}\left(S^{1}\right)
$$




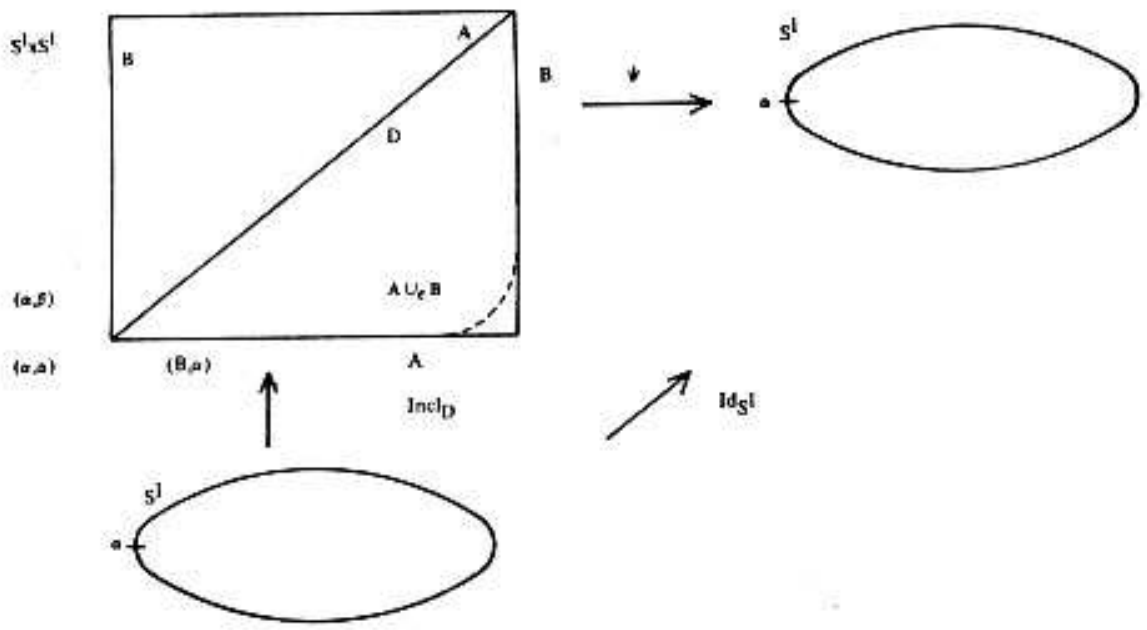

Figure 3

where $\operatorname{Incl}_{D}: S^{1} \rightarrow D$ is the inclusion map $\operatorname{Incl}_{D}(\beta)=(\beta, \beta)$, and $\operatorname{Id}\left(S^{1}\right)$ is the identity map on $S^{1}$.

Let $(\alpha, \beta)$ be a regular point of $\psi$ restricted to the set $A \cup B=\left(S^{1} \times \alpha\right) \cup$ $\left(\alpha \times S^{1}\right)$, for some $\alpha$ in $S^{1}$.

By the condition of anonymity (2),

$$
\psi(\alpha, \beta)=\psi(\beta, \alpha)
$$

which implies that for any point in $A \cup B$ that maps into a regular valuc, there must exist another point in $A \cup B$ mapping into the same value. If $A \cup B$ were smooth, this would imply that the index of the map $\psi$ restricted to $A \cup B$ is zero $(\bmod 2)$ when restricted to any regular point of $A \cup B$. However, since $A \cup B$ is not smooth, we can consider instead a smooth path, a deformation $A \cup, B$ of $A \cup B$ as indicated in Fig. 3. If $(\alpha, \beta)$ is a regular point of $\psi / A \cup_{c} B$, when $A \cup, B$ and $\psi$ are appropriately chosen smooth approximations of $A \cup B$ and of a continuous map satisfying (1), (2), (3), the index at $(\alpha, \beta)$ of $w_{i} / A \cup, B$ can be shown to be zero $(\bmod 2))^{9}$

However, since $D$ can be smoothly deformed into $A \cup_{c} B$, it follows that the index $(\bmod 2)$ of $\psi$ on $D$ should be the same as the index $(\bmod 2)$ of $\psi$ on $A \cup, B$. See, for instance, the results on index module 2 of mappings that are smoothly homotopic in [5, Chap. 5, Theorem 1.6]. Since, as we saw above,

- This can be seen by noting that for $\alpha \in S^{1}$, a a regular valuc of $\psi: A \cup, B$, we can choose an $c$ small enough that the inverse image of a under $\psi / A \cup, B$ is contained in $A \cup B$. Therefore, there will be an even number of points in the inverse image of $\alpha$, i.c., the index of $\psi / A \cup_{\mathrm{e}} B$ at $(\alpha, \beta)$ should be zero $(\bmod 2)$. 
index $\bmod 2$ of $\psi / D$ is 1 , we have a contradiction; such a smooth map cannot exist.

The above argument can be completed to provide a proof for a general casc. For instance, to complete the proof for $\psi$ continuous but not necessarily smooth, one proves that any continuous $\psi$ can be approximated arbitrarily close by a smooth map which has index $0(\bmod 2)$ on $A \cup B$ and index $1(\bmod 2)$ on $D$.

However, this argument, though intuitively appealing, does not provide the best proof since it requires that somewhat cumbersome approximation arguments be made. Instead, one can use algebraic topology tools that require only continuity of the maps, and allow the underlying spaces to be simplicial complex rather than smooth manifolds.

A simpler, if perhaps less intuitive, proof is given for $\psi$ continuous, by examining the map $\psi^{*}$ induced by $\psi$ on the $n-1$ dimensional cohomology groups of $S^{n-1}$ and $S^{n-1} \times S^{n-1}$ with $Z_{2}$ coefficients. This proof actually reproduces at the cohomology level the argument given above for the smooth case. As illustrated in Fig. 3 , in view of property (3) of $\psi$, the following diagram is commutative:

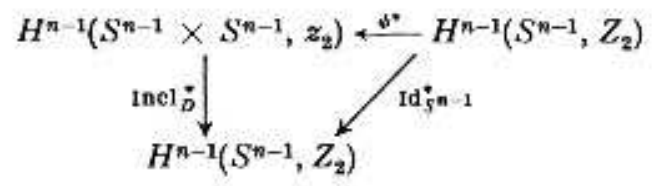

Let $G^{*}$ be a generator of $H^{n-1}\left(S^{n-1}, Z_{2}\right)$, and $A^{*}$ and $B^{*}$ the generators of $H^{n-1}\left(S^{n-1} \times S^{n-1}, Z_{2}\right) \simeq Z_{2} \times Z_{2}$ supported on $A$ and $B$, respectively. Then by (2) it must be that either

$$
\psi^{*}\left(G^{*}\right)=A^{*}+B^{*},
$$

or else

$$
\psi^{*}\left(G^{*}\right)=0(\bmod 2)
$$

since the cases when either

$$
\psi^{*}\left(G^{*}\right)=A^{*} \quad \text { or } \quad \psi^{*}\left(G^{*}\right)=B^{*}
$$

would contradict the fact that $\psi$ is symmetric on $A \cup B$, condition (2). But $\psi^{*}\left(G^{*}\right)=0$ would contradict the commutativity of the above diagram, since

$$
\operatorname{Incl}_{D}^{*} \circ \psi^{*}\left(G^{*}\right)=0 \neq G^{*}=\operatorname{Id}_{S^{n-1}}^{*}\left(G^{*}\right) .
$$

We will now check that the above diagram also contradicts $\psi^{*}\left(G^{*}\right)=A^{*}+B^{*}$. $A \cup B$ is homotopic to $D$. Hence $\operatorname{Incl}_{D}\left(S^{n-1}\right)=D$, which is homotopic to $A \cup B$, implies $\operatorname{Incl}_{D}^{*}\left(A^{*}\right)=G^{*}$, and $\operatorname{Incl}_{D}^{*}\left(B^{*}\right)=G^{*}$, and thus 
$\operatorname{Incl}_{D}^{*}\left(A^{*}+B^{*}\right)=0 \bmod 2$. This again contradicts the commutativity of the diagram, since then,

$$
\operatorname{Incl}_{D}^{*} \circ \psi^{*}\left(G^{*}\right)=0 \neq G^{*}=\mathbf{I d}_{s^{m}}^{*}\left(G^{*}\right) .
$$

This completes the proof of the case when there are two voters, i.e., $k=2$. For $k>2$ the same result obtains. The proof is analogous, studying the action of $\psi^{*}$ on the $n-1$ cohomology groups of $S^{n-1}$ and $\left(S^{n-1}\right)^{k}$ with coefficients in $Z_{k}$.

Note that in the above result it is not necessary to require that the preferences of the individuals be allowed to vary over the whole space $P$. The same result applies when the preferences of the individuals are restricted to the spaces $Q$ and $R$ of globally integrable and convex preferences, respectively, which are frequently used in economics.

Also, the results are preserved when the resulting social preference is allowed to vary over the whole space $P$. The next two corollaries follow immediately from the above result:

COROLLARY 1. Even if all individuals have globally integrable and convex preferences there does not exist a constitution $\Phi$ that assigns to an n-tuple of preferences a social preference in $P$, satisfying conditions (1), (2), and (3) of Theorem 1 .

The results of Theorem 1 and Corollary 1 prove, further:

COROLLARY 2. Neither the space of preferences $P(Q$ or $R)$ nor any continuous deformation of $P(Q$ or $R)$ admits constitutions based on additive or averaging rules. In particular, $P(Q$ or $R)$ is not a contractible space.

Proof. First we prove that contractability is sufficint for the existence of a constitution or aggregation rule satisfying conditions (1), (2), and (3) of Theorem 1.

Note that if the space of preferences at $P$ is convex, then a rule satisfying (1), (2), and (3) always exists; it is defined by

$$
\phi\left(p_{1}, \ldots, p_{k}\right)=\frac{1}{k} \sum_{i=1}^{k} p_{i}
$$

where the addition in the right-hand side is the vector addition for vector fields.

More generally, if $P$ is contractible, then $P$ is a retraction of a convex space (the space of all $C^{r}$ vector fields $V$ ) because in this case there is no topological obstruction to extending the inclusion map $i: P \rightarrow V$ to a continuous map $r: V \rightarrow V$. Since $r / p=\mathrm{id}_{P}$, it follows that $r$ is a retraction from $V$ onto $P$. 
Therefore, the social aggregation rule $\Phi$ defined by

$$
\Phi\left(p_{1}, \ldots, p_{k}\right)=r \circ\left(\frac{1}{k} \sum p_{i}\right)
$$

clearly satisfies all the required conditions. Thus any contractible space $P$ can be given an aggregation rule satisfying (1), (2), and (3). In view of Theorem I and Corollary 1 this completes the proof.

A natural question is to find out what other restrictions on $P, Q$, or $R$, other than contractibility, would assure the existence of constitutions with the desired properties. Chichilnisky [3] describes such a subspace of preferences $P$ for the case when $M$ is the unit ball in $R^{n}$, with the property that each foliation in the space $P$ admits the same global cross section, given by a one-dimensional contractability submanifold $N$ of $M$ with $\partial N C O M$. Such a subspace $P$ can be shown to admit a smooth manifold structurc, under certain regularity conditions on the foliations; see [3]. In such a subspace $\mathscr{P}$ of $P$, a constitution $\bar{\Phi}$ satisfying conditions (1), (2), and (3) exists, and it is exhibited. It is constructed using the local linear structure of $N$, since each foliation in $\mathscr{P}$ can be uniquely represented by a retraction from $M$ to $N$. The question of the nonexistence of an analogous constitution for the space of preferences $P$ becomes the nonexistence of a lifting, i.e, a continuous map $\phi$ making the following diagram commutative:

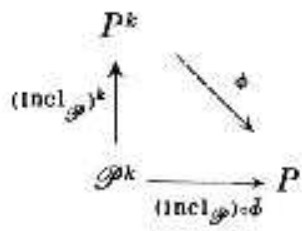

The nonexistence of such a lifting $\phi$ can be expressed as an obstruction, an element of the cohomology of $P^{k}$ relative to $y^{t}$. The study of such obstructions could give further information on the topology of $P$, and on social choice theory.

\section{REFERENCES}

1. K. Arrow, "Social Choice and Individual Values," 2nd ed. The Cowles Foundation for Research in Economics Monograph 12, Yale University Press, New Haven, Conn./London, 1963.

2. K. Arrow, "Formal Theories of Social Welfare," Reprints in Economic Theory and Econometrics No. 59, Harvard University, from "Dictionary of the History of Ideas" (P. P. Wiener, Ed.), Scribner, New York, 1973. 
3. G. Chichilnisky, "Manifolds of Preferences and Equilibria," Technical Report No. 27, Project on Efficiency of Decision Making in Economic Systems, Harvard University, 1976.

4. G. D\&HREU, Smooth preferences, Econometrica (1972).

5. M. W. Hinsch, "Differential Topology," Springer-Verlag, New YorkiHeidelberg: Berlin, 1976. 